Aus Stuart Hall wird bei Prokop Steward Hall. Hörisch verwendet zahllose fragwürdige $\mathrm{Da}$ ten und interpretiert die Abkürzung ARD zunächst als Allgemeiner Rundfunk Deutschland (Hörisch 2001, 15f.). Auch wird das Radio in Edgar Reitz' „Heimat“ nicht erst mit der Führerrede des 1. September 1939 zum Motiv, denn bereits in der ersten Folge beobachtet die Dorfgemeinschaft die Empfangsversuche von Paul Simon.

Erst die Kombination beider Publikationen mit ihren soziologischen und kulturwissenschaftlichen Erklärungsmustern ergibt ein umfassendes Gesamtspektrum der Mediengeschichte.

Joan Kristin Bleicher

\section{Urban Pappi}

\section{Teledienste, Mediendienste und Rundfunk}

Ihre Abgrenzung im Recht der elektronischen Medien

Baden-Baden: Nomos, 2000. - 176 S.

(Schriftenreihe des Archivs für Urheber- und Medienrecht; 182).

ISBN 3-7890-6954-X

\section{Babette Kibele}

\section{Multimedia im Fernsehen}

Die gesetzlichen Grundlagen audiovisueller Informations- und Kommunikationsdienste auf der Basis des deutschen und europäischen $\mathrm{Me}-$ dienrechts

München: Verlag C. H. Beck, 2001. - 258 S.

(Information und Recht; 18).

ISBN 3-406-47799-2

Die Dissertationen von Pappi (Freiburg) und Kibele (Münster) behandeln ein hochaktuelles, schwieriges und wissenschaftlich wichtiges Thema mit erheblicher Bedeutung für die Praxis: Definition und Abgrenzung von Rundfunk, Mediendiensten und Telediensten. Weitere Dissertationen zu diesem Thema sind in der Zwischenzeit veröffentlicht worden, die für die folgende Rezension jedoch nicht ausgewertet wurden; auf die Dresdener Dissertation von Claudia Bernard, Rundfunk als Rechtsbegriff, 2001 im Centaurus-Verlag veröffentlicht, und die Passauer Dissertation von Torsten Brand, Rundfunk im Sinne des Artikel 5 Abs. 1 Satz 2
GG, 2002 bei Duncker \& Humblot erschienen, sei ergänzend hingewiesen.

Die beiden vorzustellenden Arbeiten könnten unterschiedlicher kaum sein. Dies beginnt bei der äußeren Form. Während die Freiburger Dissertation (Pappi) in der UFITA-Schriftenreihe konventionell gewandet daherkommt und in der alten Rechtschreibung verfasst ist, wirkt die Arbeit von Kibele in neuer Schreibung und dem frischen Design der Schriftenreihe Information und Recht äußerlich moderner. Ferner erfreut Kibele den Leser mit einem sehr ordentlichen Stichwort- und einem eigenen Abkürzungsverzeichnis, die bei Pappi beide fehlen, was dadurch negativ verstärkt wird, dass Pappi zum Teil eigene anstelle der amtlichen Abkürzungen verwendet: $\mathrm{z}$. B. RfStV anstatt RStV für den Rundfunkstaatsvertrag oder BayRF-Gesetz statt BayRG für das Gesetz über den Bayerischen Rundfunk. Es mag grenzwertig unter Form angemerkt werden, dass Pappi in den inhaltlichen Randzonen seiner Arbeit vereinzelt unsorgfältig mit Bezeichnungen umgeht: z. B. spricht er auf S. 35 von klassischer Musik anstatt von unkommentierter Instrumentalmusik ohne Werbung, wie es in der von ihm zitierten Stelle im BayMGKommentar von Bornemann/Lörz heißt, oder er nennt die Direktorenkonferenz der Landesmedienanstalten in Fußnote 110 auf S. 33 Direktorenkonferenz der Landesmedienzentralen, was vielleicht daran liegt, dass er als Student ein Praktikum bei der Bayerischen Landeszentrale für neue Medien abgeleistet hat. Die Divergenz im Umfang der Literaturverzeichnisse (Pappi: 7 Seiten, Kibele: über 23 Seiten) ist überwiegend drucktechnisch verursacht; während durchschnittlich über 30 Werke auf jeder Seite in der Arbeit von Pappi nachgewiesen sind, enthält eine Seite im Buch von Kibele durchschnittlich weniger als 15 Werke.

Es wäre ein großer Fehler, sich nach diesem kritischen Einstieg gegen die Lektüre der Dissertation von Pappi zu entscheiden, die sich bei inhaltlicher Wertung als prägnant formulierte, scharfsinnige Arbeit der rechtswissenschaftlichen Grundlagenforschung darstellt, die auch kommunikationswissenschaftliche Erkenntnisse sinnvoll einbezieht. Demgegenüber darf der Leser bei Kibele eine im deskriptiven Teil breit angelegte Arbeit über Entwicklung und Stand von Erscheinungsformen elektronischer Informations- und Kommunikationsangebote erwarten, die jeweils die Rechtslage für den öf- 
fentlich-rechtlichen Rundfunk gesondert darstellt.

Pappi referiert - wie Kibele - die wissenschaftliche Kontroverse zur Thematik zutreffend. Der deskriptive Teil seiner Arbeit belegt, dass der Autor sich auskennt. Das erlaubt ihm, klare eigene Positionen zu beziehen. Vor eindeutig formulierter Kritik an misslungenen Regelungen schreckt er nicht zurück, etwa wenn er meint, die zersplitterte Aufsicht über die Telemedien „dürfte sich auch in der Praxis als kostenintensive Fehlkalkulation erweisen." (S. 19)

Kibele hat mit großer Sorgfalt viele Fakten zusammengetragen, die sie vollständig ausbreitet. Chronologische Darstellungen (S. 24), Begriffserklärungen (S. 31) Kategorisierungen (S. 41), Umfrageergebnisse (S. 51) oder sonstige tabellengeeignete Daten (z. B. S. 125-129, S. 211-216, 221, 231) werden durch tabellarische oder sonstige grafische Gestaltung (S. 16, $33,44,125)$ anschaulich gemacht.

Im Gegensatz zu Kibele, die im Wesentlichen die herrschende Meinung referiert, auf die vielfältigen, gut recherchierten Medienphänomene anwendet und den Gesetzgeber zu Lösungen für die erkannten Probleme aufruft, entwickelt Pappi einen eigenen rechtsdogmatischen Lösungsansatz auf der Grundlage der bestehenden Vorschriften. Dazu entfaltet er Art. 5 Abs. 1 Satz 2 GG über den Wortlaut hinaus zu einem Schutzschirm für alle massenkommunikativen Verhaltensweisen; das ermöglicht ihm eine Kategorisierung medialer Massenkommunikationsphänomene unabhängig von den Begriffen „Presse“, „Film“ und „Rundfunk“, da Art. 5 Abs. 1 Satz 2 GG nach seiner Ratio auch solche Massenkommunikation schütze, die der Verfassungsgeber noch nicht kannte und deshalb in der Aufzählung noch nicht benennen konnte. Deshalb besteht für Pappi keine Notwendigkeit, Mediendienste unter den verfassungsrechtlichen Rundfunkbegriff zu subsumieren, um ihnen den Schutz des Art. 5 Abs. 1 Satz 2 GG angedeihen zu lassen. Bekanntlich definiert das Grundgesetz den Rundfunkbegriff nicht. Die Definitionen der verschiedenen einschlägigen Gesetze (Rundfunkstaatsvertrag, Landesmediengesetze, Landesrundfunkgesetze, Bundesrundfunkgesetz) entschleiert Pappi als Anwendungsbereichsregeln, die in Wahrheit nicht den Rundfunkbegriff (mit kleinen Unterschieden) definieren, sondern den Anwendungsbereich der verschiedenen Gesetzefestlegen. Schließlich schlägt er vor, nicht allein anhand abstrakter Begriffsdefinitionen festzulegen, was im Rechtssinn Rundfunk sein soll, sondern zusätzlich anhand der Zweckbestimmung von Rundfunkrecht zu fragen, was für Rundfunk typisch ist (S. 91ff.). Dabei greift er auf die Normsituation des Erlasses des Grundgesetzes zurück: Der Verfassungsgeber hatte die bekannten Erscheinungsformen Hörfunk und Fernsehen vor Augen. Alle funktechnisch verbreiteten massenkommunikativen Medienangebote, die eine rundfunktypische Gefährdungslage für die öffentliche Meinungsbildung schaffen, bedürfen der besonderen rundfunkspezifischen gesetzlichen Ausgestaltung. Weil die weniger meinungsrelevanten Informations- und Kommunikationsangebote des Mediendienste-Staatsvertrags nach diesem Ansatz kein Rundfunk im verfassungsrechtlichen Sinn sind, sondern in Art. 5 Abs. 1 Satz 2 GG nicht ausdrücklich genannte, aber gleichwohl durch ihn grundrechtlich geschützte Massenkommunikation, entsteht das Dilemma nicht, entgegen der Doktrin des Bundesverfassungsgerichts vom verfassungsrechtlich vorgegebenen gesetzlichen Ausgestaltungsvorbehalt zulassungs- und anmeldefreie Mediendienste ohne vorgegebene Programmgrundsätze als nicht ausgestaltete Rundfunkformen verteidigen zu müssen (S. 115f.). Dass bei diesem Ansatz auch der Schönheitsfehler zweier verschiedener Rundfunkbegriffe (eines weiteren verfassungsrechtlichen und eines engeren einfachrechtlichen) vermieden wird, sei ergänzend bemerkt. Das Problem der Einord-

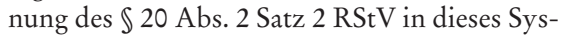
tem wird zwar gesehen, aber nicht als Problem gewertet. Bei konsequenter Anwendung des Ansatzes von Pappi wäre die Vorschrift mindestens überflüssig: Unabhängig von der Subsumtionsfähigkeit eines Dienstes unter die Regelbeispiele des $\$ 2 \mathrm{Abs} .2 \mathrm{MDStV}$ könnte im Wege der Einzelfallabwägung nach Pappis Modell immer festgestellt werden, ob ein Dienst wegen seiner (überwiegenden) typischen Merkmale Rundfunk ist. In der Tat unterscheiden sich die notwendigen Prüfungsschritte nach dem Ansatz der überwiegenden Meinung und nach Pappi nicht. Gleichwohl konstruiert der Gesetzgeber in $\mathbb{2 0}$ Abs. 2 Satz 2 RStV eine besondere Konstellation, die es nach dem auf eindeutige Ergebnisse bedachten Ansatz von Pappi gar nicht geben kann: Mediendienste, die dem Rundfunk zuzuordnen sind. Nach Pappi wären diese „Mediendienste“ aufgrund ihrer rund- 
funktypischen Merkmale bereits als Rundfunk zu definieren; die Rechtsfolge ist in beiden Modellen prinzipiell gleich. Nach Rundfunkstaatsvertrag ist jedoch eine einstimmige Entscheidung aller Landesmedienanstalten für die rechtliche Zuordnung zum Rundfunk konstitutiv. Zwar gilt dies möglicherweise nur im Hinblick auf medienrechtliche Sanktionen und bindet bereits die Gerichte in wettbewerbsrechtlichen Auseinandersetzungen nicht. Ein Dilemma liegt hierin doch: Falls das Einvernehmen unter den 15 Landesmedienanstalten nicht zustande kommt, findet Rundfunk außerhalb rundfunkrechtlicher Regeln statt (S. 153f.). Pappi löst das Problem durch Vertrauen auf „die Koordinierungsfähigkeit der Landesmedienanstalten“. (S. 154 Fn. 703) Es schmerzt den Rezensenten, dieses Vertrauen wegen der standortpolitischen Versuchungen der Landesmedienanstalten, die insoweitzum Teillandespolitischem Druck ausgesetzt sind, nicht teilen zu können. Abgesehen davon bietet Pappi ein in sich geschlossenes System an, das frei ist von logischen Brüchen und deshalb für die medienrechtliche Diskussion einen hervorragenden Beitrag zu leisten vermag.

Der nahezu aussichtlosen Debatte über die Abgrenzung von Mediendiensten und Telediensten anhand der Gesetzestexte gibt Pappi richtigerweise durch Beleuchtung mit verfassungsrechtlichen Gesetzgebungskompetenztiteln Kontur. Seine kritische Distanz zur Bundeskompetenz ist überzeugend dargelegt und schwer zu überbrücken. Mit nachvollziehbarer Schroffheit weist er die Idee zurück, Inhalte von Individualkommunikation überhaupt gesetzlich zu regeln (S. 147f.). Somit bleibt es bei einer umfassenden Länderkompetenz für die Regelung aller Erscheinungsformen der Massenkommunikation und Einzelkompetenzen des Bundes zu verschiedenen Teilbereichen (z. B. Fernmelderecht, Recht der Wirtschaft, Strafrecht, Bürgerliches Recht), die jedoch nicht als Vorwand für Gesamtregelungen von Informations- und Kommunikationsdiensten nutzbar gemacht werden dürfen. Demgegenüber bleibt Kibele auf der Ebene der Interpretation von einfachrechtlichen Gesetzestexten, denen sie versucht, das Beste abzugewinnen.

In pointierter Zusammenfassung könnte die Arbeit von Pappi als originelle rechtsdogmatische Grundlagenforschung charakterisiert werden, während Kibele mehr eine Phänomenologie aktueller medialer Angebotsformen mit historischen Reminiszenzen unter aus- drücklicher Würdigung der Vorgaben für den öffentlich-rechtlichen Rundfunk vorgelegt hat, die sauber subsumiert und in ihren rechtlichen Wertungen im Wesentlichen der überwiegenden, wenn nicht herrschenden Meinung folgt. Beide Arbeiten berücksichtigen die europarechtlichen Implikationen.

Roland Bornemann

\section{Clemens Schwender}

\section{Medien und Emotionen}

Evolutionspsychologische Bausteine einer Medientheorie

Wiesbaden: DUV, 2001. - 342 S.

ISBN 3-8244-4470-4

\section{Zugl.: Berlin, TU, Habil., 2000}

Evolutionspsychologie, Soziobiologie und Ethologie erforschen die biologischen Grundlagen menschlichen Wahrnehmens, Denkens und Verhaltens. Der Mensch ist ihnen zufolge wesentlich durch angeborene, evolutionär entwickelte Anlagen geprägt. Menschen sehen, fühlen und handeln nicht zuletzt deshalb auf bestimmte Weise, weil dies in ihrer Entwicklungsgeschichte einmal einen adaptiven Vorteil bedeutete. Letztlich sind sie wie alle Lebewesen auf das Ziel reproduktiver Fitnessmaximierung ausgerichtet. Die genannten Disziplinen gehen davon aus, dass die natürlichen Anlagen des Menschen auch sein soziokulturelles Handeln in hohem Maße beeinflussen. Wenn dies zutrifft, liegt der Gedanke nahe, evolutionspsychologische Forschungsergebnisse in die Geistes- und Sozialwissenschaften zu integrieren.

Dort stoßen die evolutionspsychologischen und soziobiologischen Thesen seit einigen Jahren auf faszinierte Zustimmung, häufiger jedoch auf scharfen Widerspruch. Denn sie treffen auf Positionen, die gerade die Freiheit des Menschen von natürlichen $Z$ wängen betonen und der Ansicht sind, dass menschliches Verhalten stärker durch andere Faktoren, etwa gesellschaftliche Kontexte, als durch angeborene Anlagen bestimmt wird. Die kulturalistischen Positionen werfen den naturalistischen Ansätzen Biologismus, Determinismus, Reduktivismus, mangelnde Falsifizierbarkeit und politische Inkorrektheit vor; die Naturalisten bezichtigen die Kulturalisten der Ignoranz gegenüber empirischen Belegen. Die Gegner stehen sich meist unversöhnlich gegenüber. 